Supplement of Geosci. Model Dev., 12, 1189-1207, 2019

https://doi.org/10.5194/gmd-12-1189-2019-supplement

(C) Author(s) 2019. This work is distributed under

the Creative Commons Attribution 4.0 License.

(c) (1)

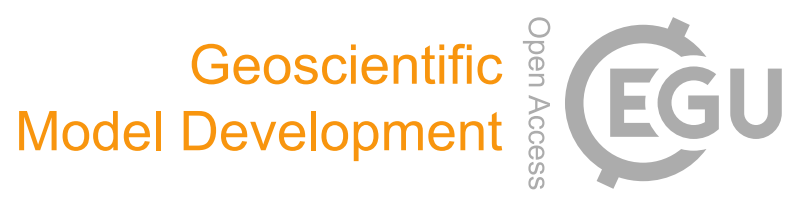

Supplement of

\title{
Discrete $k$-nearest neighbor resampling for simulating multisite precipitation occurrence and model adaption to climate change
}

\author{
Taesam Lee and Vijay P. Singh \\ Correspondence to: Taesam Lee (tae3lee@gnu.ac.kr)
}

The copyright of individual parts of the supplement might differ from the CC BY 4.0 License. 
function [DisSim] =Discrete_KNNR(BinHis, Para)

$\% \%$ Simulate Discrete Values (Binary Process) using KNNR

$\%$ Input

$\% \quad$ Para.kweight: Weight probability

$\% \quad$ Para.knum: K number

$\% \quad$ Para.Nsim: Number of simulation

$\% \quad$ Parameters required for Genertic Algorithm

$\% \quad$ Para.GA.is=true; \% if true, GA process is executed.

$\% \quad$ Para.GA.prob.cross $=0.1$; \% Crossover probability

$\% \quad$ Para.GA.prob. mut $=0.001 ; \%$ Mutation probability

$\% \quad$ BinHis: Historical Binary Data

$\%$ Output:

$\% \quad$ DisSim: number of simulation

$\mathrm{sz}=\operatorname{size}(\mathrm{BinHis})$;

$S=$ DiscrteSimWeight(Para.kweight,Para.Nsim);

BinC=BinHis(unidrnd(sz(1)),:);

SBinH=sum(BinHis(1:sz(1)-1,:),2);

Zind $H=$ find $(\sim$ SBinH $)$;nZind $H=$ length $(\mathrm{ZindH})$;

for is $=1$ :Para. Nsim

if(Para.GA.is)

set1=sel_Bin_knn(BinHis,BinC,S(is),ZindH,nZindH,sz);

if (sum(set1) $=0$ )

set2=sel_Bin_knn(BinHis,BinC,S(is),ZindH,nZindH,sz);

$\operatorname{DisSim}($ is, : $)=$ GA_Mix (set1, set2,Para,BinC);

else

$\operatorname{DisSim}($ is, : $)=$ set 1 ;

end

else

DisSim(is,: $)=$ sel_Bin_knn(BinHis,BinC,S(is),ZindH, nZindH,sz);

end

BinC=DisSim(is,:);

end

$\% \%$

function [B1] =sel_Bin_knn(BinHis,BinC,Ssel,ZindH,nZindH,sz)

$\%$ Selecting multisite occurrence from observed data

$\%$ Input:

\% BinHis: Observed Occurrence

$\%$ BinC: Current state of Occurrence

$\%$ Output:

$\% \quad$ B1: Selected Occurrence

if $\operatorname{sum}(\operatorname{Bin} C) \sim=0$ 
DistC $=\operatorname{sum}(\operatorname{abs}(\operatorname{BinHis}(1: \mathrm{sz}(1)-1,:)-\operatorname{repmat}(\operatorname{BinC}, \mathrm{sz}(1)-1,1)), 2)$;

sDistC $=$ sort(DistC);

temp $1=$ find $($ sistC $($ Ssel $)==$ DistC $)$;

B1=BinHis(temp1 (unidrnd(length(temp1)))+1,::)

else \% when all the values are zero

B1=BinHis (ZindH(unidrnd $(n Z i n d H))+1,:)$;

end

$\% \%$

function [Set_out]=GA_Mix(Set1, Set2,Para,BinC)

$\%$ GA mixing

$\%$ Input:

$\%$ Set1 and Set2 : two selected binary precipitation dataset

$\%$ BinC: Current Occrrence dataset

$\%$ Output:

$\%$ Set_out: Mixed occurrence precipitation

Set_out=Set1;

sel_cross=Para.GA.prob.cross $>$ rand(Para.N.stations, 1 );

if sum(sel_cross)

for is $=1$ :length(sel_cross)

if (sel_cross(is) \& Set2(is))

Set_out(is) $=$ Set2(is);

end

end

end

is_Mut=rand(Para.N.stations, 1)<Para.GA.prob.mut;

a $=$ find(is_Mut);

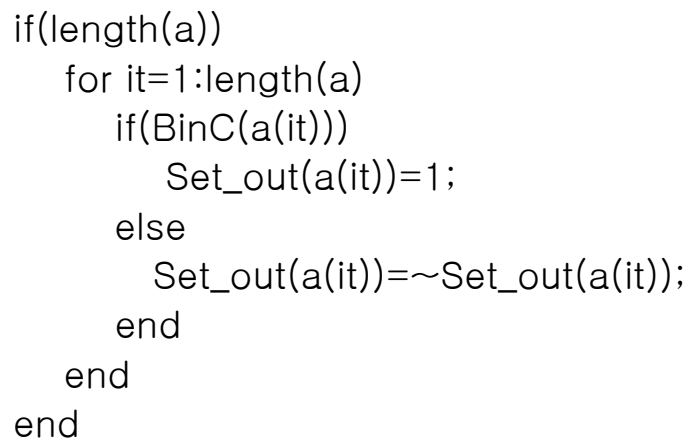

$\% \%$ Simulating Discrete Weight random number

function $S=$ DiscrteSimWeight( $p, n s)$;

$\%$ Input:

$\% \quad$ p: probability matrix

$\%$ ns: number of data to simulate 


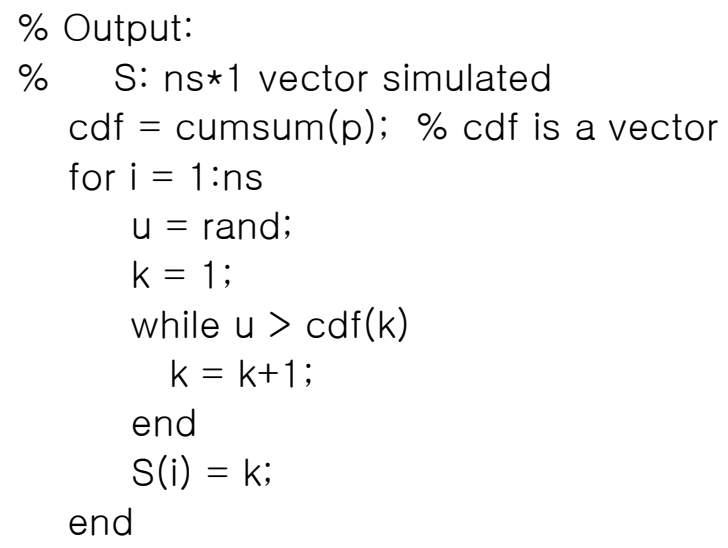

